\title{
QUANTIFICAÇÃO DA RESISTÊNCIA TÊNSIL E DA FRIABILIDADE DE UM LATOSSOLO VERMELHO DISTROFÉRRICO SOB PLANTIO DIRETO ${ }^{(1)}$
}

\author{
Cássio Antonio Tormena ${ }^{(2)}$, Marcelo Alessandro Araújo ${ }^{(3)}$, Jonez \\ Fidalski $^{(4)}$, Silvia Imhoff ${ }^{(5)}$ \& Álvaro Pires da Silva ${ }^{(6)}$
}

\begin{abstract}
RESUMO
A qualidade estrutural do solo é importante para a emergência das plântulas, bem como para o crescimento, desenvolvimento e produtividade das culturas. Uma ampla distribuição de valores de resistência tênsil do solo indica condições estruturais do solo para atender a estes pressupostos. $O$ objetivo deste trabalho foi avaliar a resistência tênsil e a friabilidade de um Latossolo Vermelho distroférrico sob plantio direto com sucessão e rotação de culturas. Os tratamentos utilizados foram: plantio direto com sucessão das culturas de trigo e soja (SDS); plantio direto com rotação de culturas, utilizando em seqüência milho-aveia-soja-aveia-soja-trigo (SDR), e SDR associado à escarificação periódica do solo (SDE). Em duas épocas distintas (outubro de 2003 e abril de 2004), foram coletados dez blocos de solo $(0,15 \times 0,20 \times 0,10 \mathrm{~m})$ em cada tratamento na camada de $0-0,20 \mathrm{~m}$ de profundidade. Quatrocentos e cinqüenta agregados de cada tratamento e época de coleta foram utilizados para determinar a resistência tênsil e a friabilidade do solo, determinando-se, também, o conteúdo de $C$ orgânico. Não foi verificada influência do C orgânico do solo na resistência tênsil e na friabilidade do solo. Verificou-se menor resistência tênsil em SDE para o ano de 2003. A sucessão de culturas (SDS) alternou temporariamente a friabilidade do solo entre a classe friável e a muito friável. A rotação de culturas (SDR) manteve temporalmente estável a estrutura
\end{abstract}

(1) Parte da Tese de Doutorado do segundo autor, apresentada ao Programa de Pós-Graduação em Agronomia da Universidade Estadual de Maringá - PGA/UEM. Recebido para publicação em abril de 2007 e aprovado em março de 2008.

${ }^{(2)}$ Professor Associado do Departamento de Agronomia da Universidade Estadual de Maringá - UEM. Av. Colombo 5790, CEP 87020-900 Maringá (PR). Bolsista do CNPq. E-mail: catormena@uem.br

(3) Engenheiro-Agrônomo, PGA/UEM. Bolsista da CAPES. E-mail: araujomaa@yahoo.com.br

(4) Pesquisador do Instituto Agronômico do Paraná - IAPAR. Caixa Postal 564, CEP 87701-970 Paranavaí (PR). E-mail: fidalski@iapar.br

(5) Professora da Universidad Nacional del Litoral. Av. P Kreder 2805, CEP 3080 Esperanza, Argentina. E-mail simhoff@unl.edu.ar

(6) Professor Titular, Departamento de Ciência do Solo, Escola Superior de Agricultura "Luiz de Queiroz" - ESALQ. Caixa Postal 09, Av. Pádua Dias 11, CEP 13418-900 Piracicaba (SP). Bolsista do CNPq. E-mail apisilva@esalq.usp.br 
do solo na classe de friabilidade do solo muito friável, assegurando melhor estrutura e qualidade física do solo. A resistência tênsil dos agregados e a friabilidade foram sensíveis na avaliação da qualidade estrutural do solo para os sistemas de manejo de solo e culturas estudados.

Termos para indexação: qualidade do solo, agregados, rotação de culturas, plantio direto, escarificação

\title{
SUMMARY: QUANTIFICATION OF TENSILE STRENGTH AND FRIABILITY OF AN OXISOL (ACRUDOX) UNDER NO-TILLAGE
}

\begin{abstract}
The soil structural quality is important for plant emergence as well as for crop growth, development and yield. A wide range of soil tensile strength values indicates that soil structure conditions meet these requirements. The objective of this study was to evaluate the tensile strength and friability of an Oxisol (Acrudox) under no-tillage with crop rotation and crop succession. The soil management treatments consisted of: crop succession with wheat and soybean (SDS); crop rotation with corn-oats-soybean-oats-soybean-wheat (SDR) and SDR plus periodic soil chiseling (SDE). Ten soil blocks $(0.15 \times 0.20 \times 0.10 \mathrm{~m})$ were randomly collected from the $0-0.20 \mathrm{~m}$ layer for each treatment, on two sampling dates (October 2003 and April 2004). The soil tensile strength and soil friability was estimated in 450 soil aggregates from each treatment and sampling date. The soil organic carbon content of the aggregates was also measured. There was no effect of soil organic carbon on soil tensile strength and friability. Results showed lower tensile strength in SDE soil collected in 2003. Crop succession (SDS) temporarily changed the friability classification from very friable to friable. Crop rotation (SDR) was effective in maintaining the soil structure at the very friable class, resulting in a better structure and soil physical quality. The parameters tensile strength and friability proved sensitive enough to evaluate soil structural quality under the crop management systems studied.
\end{abstract}

Index terms: soil quality, aggregates, crop rotation, no-tillage, chiseling.

\section{INTRODUÇÃO}

No Brasil, a área cultivada sob plantio direto é estimada em cerca de 25 milhões ha (FEBRAPDP, 2007). Neste sistema de manejo, deve-se dispor de estratégias para o controle da qualidade estrutural e física dos solos. Dentre estas, a rotação de culturas com o uso de plantas de cobertura é desejável para o manejo físico e estrutural do solo. Seus efeitos, do ponto de vista físico, podem ser refletidos na formação e estabilidade da estrutura, resultando numa ampla diversidade de tamanho de poros, com reflexos positivos na qualidade do solo e na sua sustentabilidade sob plantio direto.

Os efeitos benéficos da rotação de culturas nas propriedades físicas dos solos têm sido demonstrados pela redução na densidade do solo e aumento na porosidade (Albuquerque et al., 1995), bem como pelo controle da temperatura do solo e aumento na disponibilidade de água (Derpsch et al., 1985). No entanto, a multiplicidade dos efeitos da rotação na produtividade das culturas (Karlen et al., 1994) sinaliza a necessidade da utilização de propriedades que descrevam as modificações estruturais ocorridas na microestrutura do solo. É reconhecido que a atividade microbiana sobre a palhada e, ou, raízes resulta num contínuo fornecimento de materiais orgânicos ao solo, os quais atuam como agentes de formação e estabilização dos agregados (Campos et al., 1995; Kay \& Angers, 1999). Além disso, com a redução do revolvimento do solo em plantio direto, os ciclos de secagem e umedecimento do solo assumem importância decisiva em processos ligados à sua resiliência estrutural.

Nos solos de textura argilosa e muito argilosa, a compactação das camadas superficiais tem-se constituído numa das preocupações para o manejo do solo em plantio direto, especialmente com o uso intenso da sucessão de culturas. Nestas condições, verificase a adoção do revolvimento periódico do solo com uso de escarificação (Câmara \& Klein, 2005), na tentativa de recuperar, periodicamente, a qualidade física e estrutural do solo, sendo constatados resultados favoráveis desta prática em diversas pesquisas (Mead \& Chan, 1992; De Maria et al., 1999), sobretudo nas relativas à densidade e resistência do solo à penetração. 
Contudo, apesar de positivos, verifica-se que os efeitos da escarificação são de curta duração (Busscher et al., 1995, 2002) e podem estar associados à diminuição dos teores de matéria orgânica causada pelo favorecimento à decomposição promovida pela oxigenação temporária do solo (Araújo et al., 2004).

A qualidade física e estrutural dos solos sob plantio direto é, geralmente, avaliada por meio do estudo de propriedades ligadas à forma e à estabilidade estrutural, tais como: a densidade e a porosidade do solo (Stone \& Silveira, 2001; Bertol et al., 2004), a resistência do solo à penetração (Tormena et al., 1998) e a distribuição dos agregados em classes de tamanho ou sua estabilidade em água (Carpenedo \& Mielniczuk, 1990; Castro Filho et al., 1998). Em adição a estas propriedades, a resistência tênsil de agregados também pode ser utilizada como um indicador dos efeitos do manejo na qualidade estrutural do solo. A resistência tênsil é definida como a força por unidade de área necessária para causar o fraturamento dos agregados (Dexter \& Watts, 2000). De acordo com Dexter \& Kroesbergen (1985), a resistência tênsil é, provavelmente, a mais útil medida de resistência individual dos agregados do solo, porque pode ser determinada por um teste simples numa ampla variação de tamanhos de agregados, consistindo num indicador muito sensível da condição estrutural do solo. A magnitude da resistência tênsil reflete a hierarquia dos agregados na estrutura do solo, de modo que o fraturamento dos agregados maiores ocorre nos planos de fraqueza derivados das fissuras e microfissuras que formam zonas de menor resistência. Detalhes em relação aos métodos de medida da resistência tênsil são apresentados por Dexter \& Kroesbergen (1985), Watts \& Dexter (1998) e Dexter $\&$ Watts (2000).

A heterogeneidade da resistência tênsil resultante dos planos de fraqueza ou zonas de falhas dentro dos agregados tem sido identificada como friabilidade do solo (Dexter \& Watts, 2000). Watts \& Dexter (1998) definem friabilidade do solo como a tendência de uma massa de solo desfazer-se em tamanhos menores de agregados sob a aplicação de um estresse ou carga. De acordo com Watts \& Dexter (1998), a friabilidade do solo pode ser estimada por meio do coeficiente de variação da resistência tênsil do solo. A organização hierárquica da estrutura de um solo bem estruturado, apresentada por Dexter (1988), fornece os fundamentos do conceito da friabilidade do solo (Dexter \& Watts, 2000). Neste contexto, a friabilidade é considerada importante propriedade física dos solos agrícolas, uma vez que é desejável para o adequado estabelecimento das plantas (Snyder et al., 1995; Watts \& Dexter, 1998).

Valores elevados de friabilidade indicam que a resistência dos agregados aumenta com a redução do seu tamanho, de modo que agregados maiores poderão ser quebrados mais facilmente em menores agregados, produzindo ampla distribuição dos seus tamanhos por ocasião do preparo ou da abertura do sulco de semeadura. Macks et al. (1996) evidenciam a utilização da friabilidade como um indicador da restrição da condição estrutural do solo para o estabelecimento do plantio direto. Eles também verificaram que a friabilidade relacionava-se significativamente com outras propriedades do solo, como densidade, condutividade hidráulica e estabilidade de agregados.

Utomo \& Dexter (1981) classificaram os valores de friabilidade do solo a partir de medidas de resistência tênsil obtidas utilizando o método do volume dos agregados. Esta classificação, quando comparada com a friabilidade estimada pelo método do coeficiente de variação (Watts \& Dexter, 1998), resulta em menores valores de friabilidade. Chan et al. (1999) constataram que, para solos argilosos, ocorria uma razão média próxima a dois entre a friabilidade medida pelo método do coeficiente de variação e pelo método do volume dos agregados. Com base nestas indicações, Imhoff et al. (2002) utilizaram a classificação da friabilidade do solo proposta por Utomo \& Dexter (1981) multiplicada por dois para avaliar a qualidade estrutural de Latossolos brasileiros.

Os sistemas de manejo do solo influenciam diferentes fatores associados à resistência tênsil dos agregados e a friabilidade do solo, tais como ciclos de umedecimento e secamento (Utomo \& Dexter, 1981; Kay \& Dexter, 1992), conteúdo de água (Chan, 1989; Watts et al., 1996), dispersão e floculação das argilas (Shanmuganathan \& Oades, 1982; Kay \& Dexter, 1992; Barzegar et al., 1994), concentração e composição da solução do solo (Rahimi et al., 2000), formação e estabilidade dos agregados (Materechera et al., 1992) e teor de matéria orgânica do solo (Perfect et al., 1995; Imhoff et al., 2002). Por essa razão, a resistência tênsil dos agregados constitui uma medida sensível aos efeitos de sistemas de uso e manejo na estrutura do solo (Chan, 1995; Munkholm \& Schjønning, 2004; Blanco-Canqui et al., 2005). De acordo com Ley et al. (1993), estes efeitos podem-se refletir na qualidade estrutural do solo para as plantas, constatado pela redução no estabelecimento e crescimento de plantas em condições de elevada resistência tênsil e baixa friabilidade do solo.

Em solos tropicais e subtropicais brasileiros, tanto a resistência tênsil quanto a friabilidade não têm sido utilizadas para avaliar os efeitos de sistemas de manejo na estrutura do solo. Em solos sob plantio direto, em decorrência do reduzido revolvimento do solo e do contínuo tráfego de máquinas, as condições estruturais são controladas, basicamente, por processos naturais associados ao clima e às culturas e, eventualmente, pelo preparo periódico do solo. Dentre as práticas de manejo, a rotação de culturas exerce papel importante neste contexto. Por outro lado, o revolvimento periódico do solo com equipamentos de hastes tem sido questionado quanto à sua eficácia no manejo físico e no controle da qualidade estrutural dos solos de textura argilosa e muito argilosa. 
O objetivo do presente trabalho foi avaliar os efeitos de diferentes sistemas de manejo de solo sob plantio direto, com rotação e sucessão de culturas, na resistência tênsil de agregados e na friabilidade de um Latossolo Vermelho distroférrico.

\section{MATERIAL E MÉTODOS}

A amostragem foi realizada no município de Campo Mourão, Estado do Paraná, cuja sede situa-se $24^{\circ} 02$ ' $38^{\prime}$ " de latitude sul e $52^{\circ} 22$ ' 40 " de longitude oeste de Greenwich, apresentando relevo plano a suave ondulado, com altitude em torno de $625 \mathrm{~m}$ e com médias anuais de temperatura e precipitação pluvial de $20^{\circ} \mathrm{C}$ e $1.340 \mathrm{~mm}$, respectivamente. Nesta região, o tipo climático dominante, segundo a classificação de Köppen, é o Cfa - subtropical úmido mesotérmico (Embrapa, 1984). O solo é classificado como Latossolo Vermelho distroférrico (Neiro et al., 2003). A análise granulométrica, realizada em amostras retiradas na camada de $0-0,20 \mathrm{~m}$, indicou valores médios de $870 \mathrm{~g} \mathrm{~kg}^{-1}$ de argila, $92 \mathrm{~g} \mathrm{~kg}^{-1}$ de silte e $38 \mathrm{~g} \mathrm{~kg}^{-1}$ de areia, pertencendo o solo à classe textural muito argilosa (Embrapa, 2006).

A área experimental foi instalada em 1995 , utilizando os seguintes tratamentos de manejo: plantio direto das culturas soja-trigo em sucessão (SDS); plantio direto das culturas milho-aveia-soja-aveia-sojatrigo em rotação (SDR), e $\mathrm{SDR}$ submetido à escarificação periódica do solo numa profundidade média de 0,25 m (SDE). A escarificação foi realizada utilizando-se um escarificador com cinco hastes retas (três na barra dianteira e duas na barra traseira) munidas de ponteiras pequenas $(0,05 \mathrm{~m}$ de largura) $\mathrm{e}$ discos de corte de resíduos a frente de cada haste. As escarificações do solo, seguidas de gradagem leve a $0,08 \mathrm{~m}$, foram realizadas após a cultura de verão (milho) e antes da cultura de inverno (trigo) nos anos agrícolas 1998/1999 e 2001/2002. A correção do solo e a adubação das culturas foram feitas com base em resultados de análises de solo.

Em cada tratamento, as parcelas experimentais apresentaram dimensões de $15 \times 150 \mathrm{~m}$ foram coletados, aleatoriamente, dez blocos de solo com dimensões de 0,15 × 0,20 × $0,10 \mathrm{~m}$ na camada de 0 $0,20 \mathrm{~m}$ de profundidade, os quais foram acondicionados com filme plástico. A coleta dos blocos de solo foi realizada em duas épocas distintas: a primeira, em outubro de 2003, após a colheita da cultura de trigo, e a segunda, em abril de 2004 após a colheita da cultura de soja. No laboratório, as amostras foram manualmente destorroadas em seus agregados naturais e, em seguida, secas ao ar. No processo de quebra manual dos agregados, foi aplicada a força mínima necessária para fraturá-los em seus pontos de fraqueza preexistentes. O tamanho desejado de agregados para a determinação da resistência tênsil foi obtido por peneiramento, utilizando-se peneiras com abertura de malha entre 19,0 e 12,5 mm, conforme Imhoff et al. (2002). Para as medidas de resistência tênsil, foram separados 45 agregados em cada amostra, resultando em 450 agregados por tratamento e 1.350 agregados por época de coleta, totalizando 2.700 agregados.

O teste de tensão indireta para determinação da resistência tênsil utilizou um atuador eletrônico linear numa velocidade constante de $0,03 \mathrm{~mm} \mathrm{~s}^{-1}$, similar ao utilizado por Imhoff et al. (2002). Antes da medida, cada agregado foi pesado e, em seguida, colocado na posição mais estável para a aplicação da carga. $\mathrm{O}$ valor da carga aplicada para a quebra tênsil do agregado foi registrado por um sistema eletrônico de aquisição de dados. Após cada teste, uma alíquota dos agregados de cada amostra foi utilizada para determinação da umidade do solo; outra alíquota de agregados foi moída e tamisada em peneira com abertura de malha de $2 \mathrm{~mm}$ e, posteriormente, utilizada para a determinação do teor de $\mathrm{C}$ orgânico do solo pelo método Walkley \& Black, conforme Embrapa (1997).

A resistência tênsil (RT) foi calculada, conforme Dexter \& Kroesbergen, (1985):

$$
\mathrm{RT}=0,576\left(\frac{P}{D^{2}}\right)
$$

em que 0,576 é o coeficiente de proporcionalidade; $P$ é a força aplicada $(\mathrm{N})$, e $D$ é o diâmetro efetivo $(\mathrm{mm})$.

$\mathrm{O}$ diâmetro efetivo (D) foi calculado, conforme Watts \& Dexter (1998):

$$
\mathrm{D}=\mathrm{D}_{\mathrm{m}}\left(\frac{M}{M_{o}}\right)^{\frac{1}{3}}
$$

sendo $D_{m}$ o diâmetro médio do agregado $(\mathrm{mm}) ; M$ é a massa do agregado individual (g); e $M_{o}$ é a massa média dos agregados na população (g). O diâmetro médio dos agregados foi considerado igual à média dos tamanhos das peneiras [(19+12,5)/2] utilizadas para selecionar os agregados.

Para calcular a friabilidade do solo (F), foi utilizado o método do coeficiente de variação proposto por Watts \& Dexter (1998):

$$
F=\frac{\sigma_{\mathrm{Y}}}{\mathrm{Y}} \pm \frac{\sigma_{\mathrm{Y}}}{\mathrm{Y} \sqrt{2 n}}
$$

em que $\sigma_{\mathrm{Y}}$ é o desvio-padrão dos valores medidos da $\mathrm{RT}$; Y é a média dos valores de RT; e $n$ é o número de repetições. As classes de friabilidade utilizadas neste trabalho foram tomadas com base nos valores de $\mathrm{F}$ (adimensional) propostos por Imhoff et al. (2002): nãofriável $(<0,01)$, ligeiramente friável $(0,10-020)$, friável $(0,20-0,50)$, muito friável $(0,50-0,80)$ e mecanicamente instável $(>0,80)$. 
Os valores originais das variáveis foram submetidos às análises de variância e teste para a comparação de médias utilizando-se o delineamento inteiramente casualizado com três tratamentos (SDS, SDR e SDE) e 10 repetições (blocos de solo). As análises de variância atenderam às pressuposições básicas de distribuição normal dos resíduos pelo teste ShapiroWilk e homocedasticidade das variâncias pelo teste de Levene (Vieira, 1999), ambos com probabilidades superiores a $3 \%$. As análises estatísticas foram realizadas com o programa estatístico SAS (SAS, 2001).

\section{RESULTADOS E DISCUSSÃO}

Os valores de C orgânico do solo, teor de água dos agregados e friabilidade apresentaram pequena amplitude de variação entre os tratamentos, independentemente da época de amostragem (Quadro 1). Não foram constatadas diferenças nos teores de C orgânico do solo entre os tratamentos ( $p>0,05)$, o que pode ser atribuído ao efeito de longo prazo do plantio direto na sua homogeneização na camada amostrada. O teor de água dos agregados, por ocasião da medida da RT, não foi significativamente alterado pelos tratamentos, indicando que a variação da RT e a F entre os tratamentos deveu-se exclusivamente aos efeitos destes na estrutura do solo.

A RT apresentou valores elevados de coeficiente de variação, superiores aos descritos por Dexter \& Kroesbergen (1985) e por Imhoff et al. (2002). De acordo com Imhoff et al. (2002), um dos fatores que influencia a RT é a forma dos agregados. Neste estudo, verificou-se grande variação no formato dos agregados, desde formas mais esféricas até formas

Quadro 1. Momentos estatísticos para variáveis de solo determinadas em agregados de um Latossolo Vermelho distroférrico submetido a diferentes sistemas de manejo: sucessão de culturas (SDS), rotação de culturas (SDR) e rotação de culturas com escarificação-(SDE), em 2003 e 2004

\begin{tabular}{|c|c|c|c|c|c|}
\hline Variável & Média & Desvio-padrão & CV (\%) & Mínimo & Máximo \\
\hline \multicolumn{6}{|c|}{ SDS em 2003} \\
\hline Carbono orgânico ( $\left.\mathrm{g} \mathrm{kg}^{-1}\right)$ & 22,77 & 1,510 & 6,650 & 20,75 & 25,14 \\
\hline Teor de água (kg kg·1) & 0,027 & 0,003 & 11,11 & 0,024 & 0,033 \\
\hline Resistência tênsil (kPa) & 89,35 & 39,50 & 44,21 & 15,10 & 287,4 \\
\hline Friabilidade (1) & 0,420 & 0,070 & 15,91 & 0,340 & 0,540 \\
\hline \multicolumn{6}{|c|}{ SDS em 2004} \\
\hline Carbono orgânico (g kg-1) & 23,42 & 2,400 & 10,23 & 17,56 & 25,94 \\
\hline Teor de água $\left(\mathrm{kg} \mathrm{kg}^{-1}\right)$ & 0,029 & 0,001 & 3,4482 & 0,027 & 0,031 \\
\hline Resistência tênsil (kPa) & 83,20 & 46,61 & 56,02 & 17,96 & 435,6 \\
\hline Friabilidade (1) & 0,490 & 0,110 & 22,29 & 0,350 & 0,660 \\
\hline \multicolumn{6}{|c|}{ SDR em 2003} \\
\hline Carbono orgânico (g kg-1) & 22,76 & 2,620 & 11,49 & 19,15 & 26,33 \\
\hline Teor de água $\left(\mathrm{kg} \mathrm{kg}^{-1}\right)$ & 0,027 & 0,002 & 7,407 & 0,025 & 0,036 \\
\hline Resistência tênsil (kPa) & 97,20 & 53,89 & 55,44 & 19,77 & 321,79 \\
\hline Friabilidade ${ }^{(1)}$ & 0,520 & 0,070 & 12,84 & 0,450 & 0,650 \\
\hline \multicolumn{6}{|c|}{ SDR em 2004} \\
\hline Carbono orgânico (g kg-1) & 22,10 & 2,590 & 11,73 & 19,15 & 25,94 \\
\hline Teor de água (kg kg-1) & 0,025 & 0,003 & 13,00 & 0,021 & 0,029 \\
\hline Resistência tênsil (kPa) & 86,44 & 40,93 & 47,35 & 25,49 & 288,48 \\
\hline Friabilidade & 0,480 & 0,050 & 10,15 & 0,420 & 0,560 \\
\hline \multicolumn{6}{|c|}{ SDE em 2003} \\
\hline Carbono orgânico ( $\left.\mathrm{g} \mathrm{kg}^{-1}\right)$ & 24,34 & 1,030 & 4,230 & 23,14 & 26,33 \\
\hline Teor de água $\left(\mathrm{kg} \mathrm{kg}^{-1}\right)$ & 0,028 & 0,002 & 7,142 & 0,026 & 0,032 \\
\hline Resistência tênsil (kPa) & 83,88 & 44,90 & 53,52 & 14,36 & 303,3 \\
\hline Friabilidade ${ }^{(1)}$ & 0,530 & 0,060 & 12,16 & 0,450 & 0,650 \\
\hline \multicolumn{6}{|c|}{ SDE em 2004} \\
\hline Carbono orgânico (g kg-1) & 24,04 & 1,290 & 5,350 & 21,55 & 26,33 \\
\hline Teor de água $\left(\mathrm{kg} \mathrm{kg}^{-1}\right)$ & 0,029 & 0,002 & 6,896 & 0,024 & 0,031 \\
\hline Resistência tênsil (kPa) & 88,18 & 42,20 & 47,84 & 19,36 & 265,1 \\
\hline Friabilidade ${ }^{(1)}$ & 0,470 & 0,080 & 16,07 & 0,340 & 0,600 \\
\hline
\end{tabular}

\footnotetext{
(1) Friável $(0,20<\mathrm{F}<0,50)$ e muito friável $(0,50<\mathrm{F}<0,80)$.
} 
mais prismáticas, o que pode justificar a elevada variabilidade das medidas da RT. Ressalta-se que o solo estudado apresenta mineralogia composta por caulinita e óxidos de $\mathrm{Fe}$ e $\mathrm{Al}$ (Costa, 1996), determinando uma estrutura dominada por agregados arredondados e com elevada porosidade, especialmente considerando os elevados teores de C do solo (Quadro 1). No entanto, as variações no formato e, por conseqüência, na RT dos agregados podem ser associadas não só a interferências na estrutura causadas por pressões aplicadas ao solo pelas máquinas, pela ação dos sistemas radiculares das culturas, mas também à heterogeneidade espacial e temporal dos ciclos de umedecimento e secagem que ocorrem nas posições de linha e entrelinha das plantas, como verificado por Zhai et al. (1990).

Os teores de C orgânico não apresentaram correlação significativa com a RT e F do solo ( $p>0,05)$, em concordância com os resultados de Imhoff et al. (2002). Estes resultados diferem dos obtidos por Macks et al. (1996) e Watts \& Dexter (1998) que, provavelmente, em razão da utilização de solos com faixa mais ampla de teor de C orgânico, obtiveram correlação significativa destas variáveis com teor de $\mathrm{C}$ orgânico do solo. Da mesma forma, não foi verificada correlação significativa de RT e F com o teor de água do solo, o que difere dos resultados de Utomo \& Dexter (1981) e Perfect et al. (1995), mas corrobora as observações de Imhoff et al. (2002) e Giarola et al. (2003) em solos tropicais com mineralogia similar. O teor de água dos agregados durante a determinação de RT foi em média menor que $0,03 \mathrm{~kg} \mathrm{~kg}^{-1}$, o que se traduz num detalhe metodológico importante. De acordo com Dexter \& Watts (2000), agregados mais úmidos podem sofrer uma deformação plástica antes de ocorrer a quebra tênsil do agregado, levando à incorreta determinação da RT.

Os valores do parâmetro W de Shapiro-Wilk e a probabilidade associada (p) para as variáveis $\mathrm{RT}$ e $\mathrm{F}$ foram, respectivamente, maiores que 0,95 e 0,05 , independentemente do tratamento e época de amostragem. A RT apresentou distribuição lognormal (Figura 1). Estes resultados equivalem aos de Giarola et al. (2003), Imhoff et al. (2002) e Perfect et al. (1995). No entanto, os resultados de Bartoli et al. (1992) e Dexter \& Watts (2000) indicaram a distribuição normal para RT, diferindo dos resultados obtidos neste estudo. No tratamento SDE, ocorreu maior freqüência de elevados valores de RT no ano de 2004 e menores em 2003 (Figura 1). Nos tratamentos SDR e SDS, esta variação na distribuição de RT entre os anos de coleta não foi tão bem definida quanto em SDE, provavelmente por estar associada ao efeito residual da escarificação do solo.

Na primeira época de coleta (2003), o tratamento SDR apresentou maior valor de RT e diferiu estatisticamente $(p<0,10)$ do tratamento SDE que apresentou o menor valor de RT (Figura 2). Valores menores de RT no tratamento SDE podem ser atribuídos ao efeito residual da escarificação, ocorrida cerca de um ano antes da coleta dos agregados. No tratamento SDR, o maior valor de RT pode estar associado à presença de agregados mais estáveis no solo, resultante da maior diversidade e atividade de raízes das diferentes culturas em rotação. A maior RT em SDR pode assegurar maior estabilidade do sistema poroso aos diferentes estresses aplicados ao solo $\mathrm{e}$, indiretamente, torna-se benéfica às plantas. Tormena et al. (2007), em estudo realizado na mesma área, verificaram maiores valores médios de densidade do solo nos tratamentos SDR e SDS, os quais indicaram a presença de agregados individuais com maiores valores de densidade e, por conseqüência, de RT. A redução na densidade do solo implicou redução da RT, como atestado por Zhang (1994) e Barral et al. (1998). Já na segunda época de coleta (2004), não houve diferença de RT entre os tratamentos $(p>0,10)$.

Os valores médios de RT encontrados neste estudo foram superiores àqueles verificados por Imhoff et al. (2002) e Giarola et al. (2003). Estes resultados podem

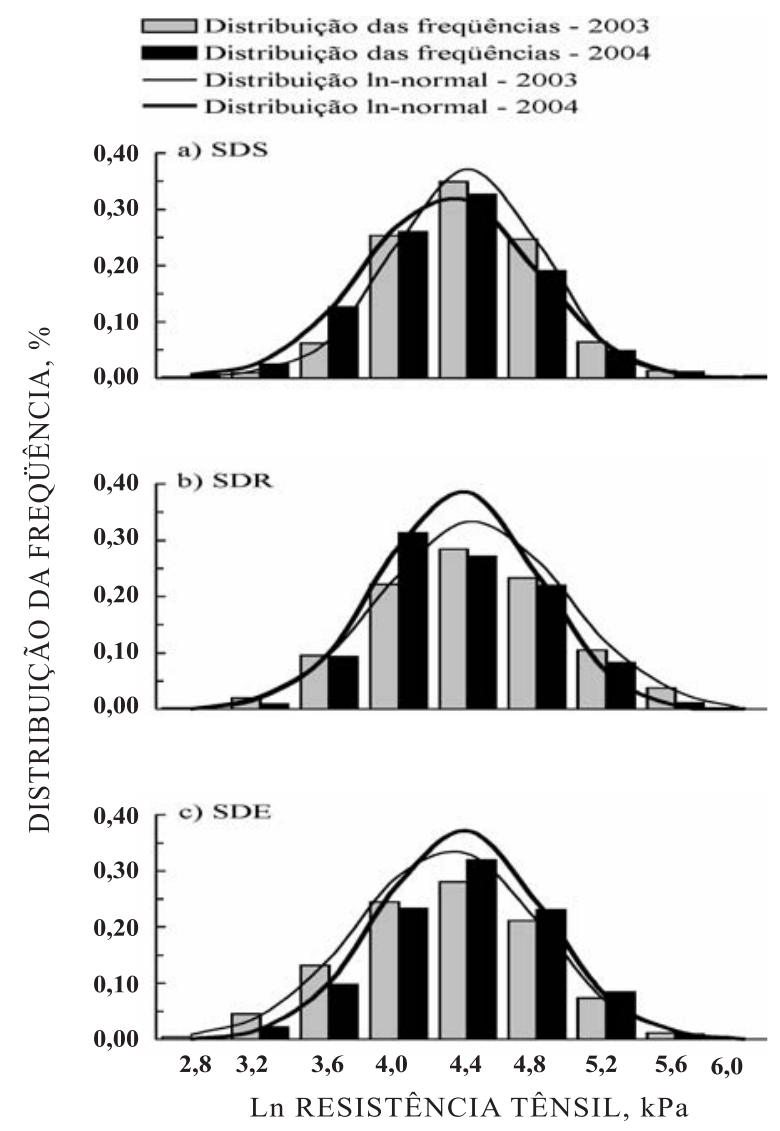

Figura 1. Freqüência da distribuição e distribuição ln-normal da resistência tênsil de agregados de um Latossolo Vermelho distroférrico submetido a diferentes sistemas de manejo do solo: a) sucessão de culturas - SDS; b) rotação de culturas - SDR, e c) rotação de culturas com escarificação - SDE, em 2003 e 2004. 


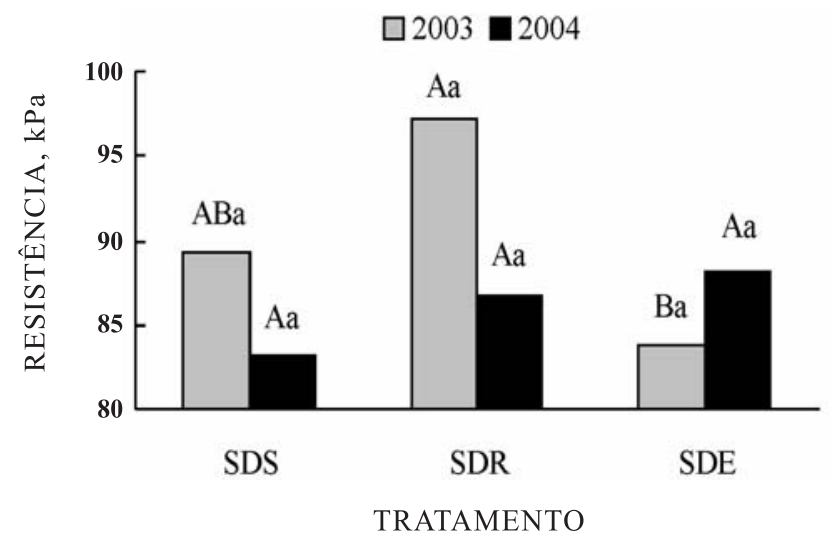

Figura 2. Valores médios de resistência tênsil de agregados de um Latossolo Vermelho distroférrico submetido a diferentes sistemas de manejo do solo: sucessão de culturas (SDS), rotação de culturas (SDR), e rotação de culturas com escarificação-(SDE), em 2003 e 2004. Letras maiúsculas comparam os tratamentos dentro do mesmo ano, e letras minúsculas comparam os anos dentro do mesmo tratamento. Médias seguidas pela mesma letra não diferem entre si pelo teste Tukey a $10 \%$.

estar relacionados com os elevados teores de argila $\left(870 \mathrm{~g} \mathrm{~kg}^{-1}\right)$ e C orgânico do solo (Quadro 1). Neste sentido, os resultados de Bartoli et al. (1992), para Latossolos (Oxissolos) provenientes do Brasil e da Índia, indicaram maiores valores de RT nos solos que apresentaram maior percentagem de argila em associação com o maior teor de C orgânico, atribuindo este resultado ao efeito agregador da matéria orgânica na estrutura do solo. Outros autores também constataram que solos com elevados teores de argila tendem a apresentar maiores valores de RT (Kemper et al., 1987; Guérif, 1990; Materechera et al., 1992; Imhoff et al., 2002).

Com relação às épocas das coletas, não foram verificadas diferenças significativas de RT entre os tratamentos (Figura 2). Para o tratamento SDE, estes resultados estão de acordo com os de Busscher et al. (1995, 2002), indicando que o efeito do preparo mecânico com equipamento de hastes é temporário e a reconsolidação do solo aumenta com o volume cumulativo de chuva. O comportamento da RT em SDE (Figura 2) também pode estar relacionado com o fenômeno que Dexter (1988) definiu como "agehardening" ou "recuperação da resistência". Este fenômeno tem como base o fato de que as ligações entre as partículas do solo que foram quebradas em decorrência da escarificação tendem a ser reconstituídas com o tempo. Os mecanismos envolvidos no fenômeno relacionam-se com o rearranjo das partículas do solo via processos de floculação das partículas de argila, com as modificações na distribuição do tamanho de poros e com a recuperação das ligações cimentantes entre as partículas do solo, refletindo em aumento da RT.

$\mathrm{Na}$ amostragem realizada em 2003, o tratamento SDS apresentou $\mathrm{F}$ significativamente menor do que os demais tratamentos $(p<0,10)$ (Figura 3). De acordo com a classificação utilizada por Imhoff et al. (2002), na amostragem realizada em 2003, os tratamentos foram enquadrados nas seguintes classes de friabilidade: SDS (friável), SDR e SDE (friável a muito friável) e, na segunda amostragem (2004), todos os tratamentos enquadraram-se na classe de friável a muito friável. Estes resultados estão em consonância com os de Imhoff et al. (2002) que, analisando o resultado de 25 amostras provenientes de três Latossolos diferentes, constataram que elas receberam a classificação "friável" e "muito friável". O tratamento SDS apresentou o menor valor de $\mathrm{F}$ em 2003 , indicando perda da qualidade estrutural e física do solo em comparação com SDR e SDE (Figura 3). Estes resultados mostram que, por ocasião da semeadura, ocorreu menor amplitude na distribuição de tamanho dos agregados no solo sob SDS, acarretando perda da qualidade física do solo para a emergência das plântulas. Apesar dos maiores valores médios de RT, não se verificou redução em $\mathrm{F}$ no tratamento SDR (Figuras 2 e 3). Segundo Dexter (2004), a RT e a F do solo são controladas pela distribuição das microfissuras e pontos de fraqueza no interior dos agregados do solo. Assim, os resultados no SDR podem estar associados às variações na intensidade e freqüência dos ciclos de secagem e umedecimento do solo, considerando as diferentes culturas em seqüência neste tratamento, resultando em agregados mecanicamente mais fortes, mas que, sob aplicação de uma carga, produzem ampla distribuição de tamanhos de agregados. A similaridade nos valores de F indica que a escarificação pode ser substituída pela rotação de culturas como prática de manejo físico do solo, com a vantagem de produzir um meio de semeadura com condições físicas e, provavelmente, biológicas mais favoráveis às plantas.

Na segunda coleta (2004), não foram constatadas diferenças significativas $(\mathrm{p}<0,10)$ para $\mathrm{F}$ entre os tratamentos (Figura 3), visto que os tratamentos estiveram sob a influência da mesma cultura (trigo), o que, em curto prazo, promoveu a homogeneização na estrutura do solo. A ocorrência de menores valores de $\mathrm{F}$ indica que o solo pode ser fraturado em fragmentos de tamanho arbitrário (muito grandes e muito pequenos), quando submetido a qualquer ação mecânica (semeadura), resultando em um meio físico inadequado para a germinação e crescimento das plântulas. Por outro lado, valores excessivamente elevados de friabilidade indicam a possibilidade de o solo sofrer intenso fraturamento, quando uma mínima força é aplicada, razão pela qual é imprópria sua utilização em culturas agrícolas mecanizadas (Utomo \& Dexter, 1981; Macks et al., 1996). Em solos sob plantio direto, a condição de solo "friável" é importante 




Figura 3. Valores médios da friabilidade do solo (limites inferiores e superiores indicados pelas barras verticais) determinados em agregados de um Latossolo Vermelho distroférrico submetido a diferentes sistemas de manejo do solo: sucessão de culturas (SDS), rotação de culturas (SDR), e rotação de culturas com escarificação -(SDE), em 2003 e 2004. Letras maiúsculas comparam os tratamentos dentro do mesmo ano e letras minúsculas comparam os anos dentro do mesmo tratamento. Médias seguidas pela mesma letra não diferem entre si pelo teste Tukey a $10 \%$ de probabilidade. Friabilidade do solo: friável $(0,20<\mathrm{F}<0,50)$ e muito friável $(0,50<\mathrm{F}<0,80)$.

para que ocorra a produção de um leito de semeadura adequado que assegure ótimas condições de germinação e estabelecimento das plantas com o mínimo de movimentação do solo, conforme atestam os resultados de Macks et al. (1996).

Neste estudo, todos os tratamentos enquadram-se dentro da classe de friável até muito friável, evidenciando a existência de condições físicas adequadas para bom contato solo-semente por ocasião da semeadura. No entanto, a maior variabilidade de RT e F em SDS, verificada nas amostragens (Figuras 2 e 3), revela menor estabilidade estrutural do solo do ponto de vista físico-mecânico, condicionando maior variabilidade temporal e espacial da estrutura do solo e da performance das culturas, dependendo das condições climáticas.

Os resultados deste trabalho indicam que a rotação de culturas (SDR) levou à ocorrência de valores elevados de RT dos agregados. No entanto, a hierarquia e a organização estrutural do solo sob SDR permitiram uma variabilidade de valores de RT compatível com uma condição de friabilidade na classe de friável a muito friável. Apesar de a prática de escarificação ter sido efetiva para esse fim, SDE não resultou em ganho de qualidade estrutural do solo comparado ao SDR. Neste sentido, ressalta-se que a adoção de um sistema planejado de rotação de culturas (SDR) é imprescindível para a qualidade estrutural do solo em plantio direto e, na condição deste solo e condições de manejo, implica suprimir a escarificação do solo como prática mecânica complementar de manejo físico do solo.

\section{CONCLUSÕES}

1. Não foi verificada influência do $\mathrm{C}$ orgânico do solo na resistência tênsil e na friabilidade do solo, enquanto a escarificação apresentou efeito efêmero sobre a resistência tênsil do solo estudado.

2. A sucessão de culturas reduziu temporalmente a friabilidade do solo, com alternância entre a classe friável e a muito friável.

3. A rotação de culturas manteve temporalmente estável a estrutura do solo na classe de friabilidade muito friável, assegurando a estrutura e a qualidade física do solo.

4. A resistência tênsil dos agregados e a friabilidade mostraram-se como indicadores sensíveis na avaliação da qualidade estrutural do solo nos tratamentos estudados.

\section{LITERATURA CITADA}

ALBUQUERQUE, J.A.; REINERT, D.J.; FIORIN, J.E.; RUEDELL, J.; PETRERE, C. \& FONTINELLI, F. Rotação de culturas e sistemas de manejo do solo: Efeito sobre a forma da estrutura do solo ao final de sete anos. R. Bras. Ci. Solo, 19:115-119, 1995.

ARAÚJO, M.A.; TORMENA, C.A.; INOUE, T.T. \& COSTA, A.C.S. Efeitos da escarificação na qualidade física de um Latossolo Vermelho distroférrico após treze anos de semeadura direta. R. Bras. Ci. Solo, 28:495-504, 2004.

BARRAL, M.T.; ARIAS, M. \& GUÉRIF, J. Effects of iron and organic matter on the porosity and structural stability of soil aggregates. Soil Till. Res., 46:261-272, 1998.

BARTOLI, F.; BURTIN, G. \& GUÉRIF, J. Influence of organic matter on aggregation in Oxisols rich in gibbsite or in goethite. II. Clay dispersion, aggregate strength and water-stability. Geoderma, 54:259-274, 1992.

BARZEGAR, A.R.; MURRAY, R.S.; CHURCHMAN, G.J. \& RENGASAMY, P. The strength of remolded soils as affected by exchangeable cations and dispersible clay. Aust. J. Soil Res., 32:185-199, 1994.

BERTOL, I.; ALBUQUERQUE, J.A.; LEITE, D.; AMARAL, A.J. \& ZOLDAN JUNIOR, W.A. Propriedades físicas do solo sob preparo convencional e semeadura direta em rotação e sucessão de culturas, comparadas às do campo nativo. R. Bras. Ci. Solo, 28:155-163, 2004.

BLANCO-CANQUI, H.; LAL, R.; OWENS, L.B.; POST, W.M. \& IZAURRALDE, R.C. Mechanical properties and organic carbon of soil aggregates in the Northern Appalachians. Soil Sci. Soc. Am. J., 69:1472-1481, 2005. 
BUSSCHER, W.J.; BAUER, P.J. \& FREDERICK, J.R. Recompaction of a coastal loamy sand after deep tillage as a function of subsequent cumulative rainfall. Soil Till. Res., 68:49-57, 2002.

BUSSCHER, W.J.; EDWARDS, J.H.; VEPRASKAS, M.J. \& KARLEN, D.L. Residual effects of slit tillage and subsoiling in a hardpan soil. Soil Till. Res., 35:115-123, 1995.

CÂMARA, R.K. \& KLEIN, V.A. Propriedades físico-hídricas do solo sob plantio direto escarificado e rendimento da soja. Ci. Rural, 35:813-819, 2005.

CAMPOS, B.C.; REINERT, D.J.; NICOLODI, R.; RUEDELL, J. \& PETRERE, C. Estabilidade estrutural de um Latossolo Vermelho-Escuro distrófico após sete anos de rotação de culturas e sistemas de manejo do solo. R. Bras.Ci. Solo, 19:121-126, 1995.

CARPENEDO, V. \& MIELNICZUK, J. Estado de agregação e qualidade de agregados de Latossolos Roxos, submetidos a diferentes sistemas de manejo. R. Bras. Ci. Solo, 14:99$105,1990$.

CASTRO FILHO, C.; MUZILLI, O. \& PODANOSCHI, A.L Estabilidade dos agregados e sua relação com o teor de carbono orgânico num Latossolo Roxo distrófico, em função de sistemas de plantio, rotações de culturas e métodos de preparo das amostras. R. Bras. Ci. Solo, 22:527538, 1998.

CHAN, K.Y. Friability of a hardsetting soil under different tillage and land use practices. Soil Till. Res., 13:287-298, 1989.

CHAN, K.Y. Strength characteristics of a potentially hardsetting soil under pasture and conventional tillage in the semi-arid region of Australia. Soil Till. Res., 34:105$113,1995$.

CHAN, K.Y.; DEXTER, A.R. \& MCKENZIE, D.C. Categories of soil structure base on mechanical behavior and their evaluation using additions of lime and gypsum on a sodic Vertisol. Aust. J. Soil Res., 37:903-911, 1999.

COSTA, A.C.S. Iron oxide mineralogy of soils derived from volcanic rocks in the Parana River Basin, Brazil. Columbus, The Ohio State University, 1996. 243p. (Tese de Doutorado)

DE MARIA, I.C.; CASTRO, O.M. \& SOUZA DIAS, H. Atributos físicos do solo e crescimento radicular de soja em Latossolo Roxo sob diferentes métodos de preparo do solo. R. Bras. Ci. Solo, 23:703-709, 1999.

DERPSCH, R.; SIDIRAS, N. \& HEINZMANN, F.X. Manejo do solo com coberturas verdes de inverno. Pesq. Agropec. Bras., 20:761-773, 1985.

DEXTER, A.R. Advances in characterization of soil structure. Soil Till. Res., 11:199-238, 1988.

DEXTER, A.R. \& KROESBERGEN, B. Methodology for determination of tensile strength of soil aggregates. J. Agric. Eng. Res., 31:139-147, 1985.
DEXTER, A.R. \& WATTS, C. Tensile strength and friability. In: SMITH, K. \& MULLINS, C., eds. Soil and environmental analysis: Physical methods. 2.ed. New York, Marcel Dekker, 2000. p.405-433.

DEXTER, A.R. Soil physical quality: Part I. Theory, effects of soil texture, density, and organic matter, and effects on root growth. Geoderma, 120:201-214, 2004.

EMPRESA BRASILEIRA DE PESQUISA AGROPECUÁRIA EMBRAPA. Centro nacional de Pesquisa de Solos. Manual de métodos de análises de solo. 2.ed. Rio de Janeiro, 1997. 212p.

EMPRESA BRASILEIRA DE PESQUISA AGROPECUÁRIA EMBRAPA. Serviço Nacional de Levantamento e Conservação de Solos. Levantamento de reconhecimento dos solos do Estado do Paraná. Londrina, EmbrapaSNLCS/SUDESUL/IAPAR, 1984. v.1/2, 791p. (EmbrapaSNLCS. Boletim de Pesquisa, 27; IAPAR. Boletim Técnico, 16)

EMPRESA BRASILEIRA DE PESQUISA AGROPECUÁRIA . EMBRAPA. Sistema brasileiro de classificação de solos. 2.ed. Rio de Janeiro, 2006. 306p.

FEDERAÇÃO BRASILEIRA DE PLANTIO DIRETO NA PALHA - FEBRAPDP. Área de plantio direto no Brasil. Disponível em <http://www.febrapdp.org.br/port/plantio direto $>$. Acesso em: 10 de maio de 2007.

GIAROLA, N.F.B.; SILVA, A.P.; IMHOFF, S. \& DEXTER, A.R. Contribution of natural soil compaction on hardsetting behavior. Geoderma, 113:95-108, 2003.

GUÉRIF, J. Factors influencing compaction-induced increases in soil strength. Soil Till. Res., 16:167-178, 1990.

IMHOFF, S.; SILVA, A.P. \& DEXTER, A.R. Factors contributing to the tensile strength and friability of Oxisols. Soil Sci. Soc. Am. J., 66:1656-1661, 2002.

KARLEN, D.L.; VARVEL, G.E.; BULLOCK, D.G. \& CRUSE, R.M. Crop rotations for the $21^{\text {st }}$ century. Adv. Agron., 53:1-45, 1994.

KAY, B.D. \& ANGERS, D.A. Soil structure. In: SUMNER, M.E., ed. Handbook of soil science. Boca Raton, CRC Press, 1999. p.229-276.

KAY, B.D. \& DEXTER, A.R. The influence of dispersible clay and wetting/drying cycles on the tensile strength of a Red-Brown Earth. Aust. J. Soil Res., 30:297-310, 1992.

KEMPER, W.D.; ROSENAU, R.C. \& DEXTER, A.R. Cohesion development in disrupted soils as affected by clay and organic matter content and temperature. Soil Sci. Soc. Am. J., 51:860-867, 1987.

LEY, G.J.; MULLINS, C.E. \& LAL, R. Effects of soil properties on the strength of weakly structures tropical soils. Soil Till. Res., 28:1-13, 1993.

MACKS, S.P.; MURPHY, B.W.; CRESSWELL, H.P. \& KOEN, T.B. Soil friability in relation to management history and suitability for direct drilling. Aust. J. Soil Res., 34:343360, 1996 .

MATERECHERA, S.A.; DEXTER, A.R. \& ALSTON, A.M. Formation of aggregates by plant roots in homogenized soils. Plant Soil, 142:69-79, 1992. 
MEAD, J.A. \& CHAN, K.Y. Cultivation techniques and grazing affect surface structure of an Australian hardsetting soil. Soil Till. Res., 25:217-230, 1992.

MUNKHOLM, L.J. \& SCHJØNNING, P. Structural vulnerability of a sandy loam exposed to intensive tillage and traffic in wet conditions. Soil Till. Res., 79:79-85, 2004.

NEIRO, E.S.; MATA, J.D. V.; TORMENA, C.A.; GONÇALVES, A.C.A.; PINTRO, J.C. \& COSTA, J.M. Resistência à penetração de um Latossolo Vermelho distroférrico, com rotação e sucessão de culturas, sob plantio direto. Acta Sci., 25:19-25, 2003.

PERFECT, E.; KAY, B.D. \& SILVA, A.P. Influence of soil properties on the statistical characterization of dry aggregate strength. Soil Sci. Soc. Am. J., 59:532-537, 1995.

RAHIMI, H.; PAZIRA, E. \& TAJIK, F. Effect of soil organic matter, electrical conductivity and sodium adsorption ratio on tensile strength of aggregates. Soil Till. Res., 54:145$153,2000$.

SAS Institute. SAS/STAT user's guide. Version 8.2. Cary, 2001. $943 p$.

SHANMUGANATHAN, R.T. \& OADES, J.M. Effect of dispersible clay on the physical properties of the B horizon of a Red-brown Earth. Aust. J. Soil Res., 20:315-324, 1982

SNYDER, V.A.; VÁZQUEZ, M.A.; MARTÍNEZ, G.; RAMÍREZ, L. \& HADAS, A. Controlled displacement technique for measuring soil friability. Soil Sci. Soc. Am. J., 59:44-52, 1995.
STONE, L.F. \& SILVEIRA, P.M. Efeitos do sistema de preparo e da rotação de culturas na porosidade e densidade do solo. R. Bras. Ci. Solo, 25:395-401, 2001.

TORMENA, C.A.; ARAÚJO, M.A.; FIDALSKI, J. \& COSTA, J.M. Variação temporal do intervalo hídrico ótimo de um Latossolo Vermelho distroférrico sob sistemas de plantio. R. Bras. Ci. Solo, 31:211-219, 2007.

TORMENA, C.A.; SILVA, A.P. \& LIBARDI, P.L. Caracterização do intervalo hídrico ótimo de um Latossolo Roxo sob plantio direto. R. Bras. Ci. Solo, 22:573-581, 1998.

UTOMO, W.H. \& DEXTER, A.R. Soil friability. J. Soil Sci., 32:203-213, 1981

VIEIRA, S. Pressuposiçoes básicas. In: VIEIRA, S. Estatística experimental. 2.ed. São Paulo, Atlas, 1999. p.133-147.

WATTS, C.W. \& DEXTER, A.R. Soil friability: Theory, measurement and the effects of management and organic carbon content. Eur. J. Soil Sci., 49:73-84, 1998.

WATTS, C.W.; DEXTER, A.R. \& LONGSTAFF, D.J. An assessment of the vulnerability of soil structure to destabilisation during tillage. Part II. Field trials. Soil Till. Res., 37:175-190, 1996.

ZHAI, R.; KACHANOSKI, R.G. \& VORONEY, R.P. Tillage effects on the spatial and temporal variations of soil water. Soil Sci. Soc. Am. J., 54:186-192, 1990.

ZHANG, H. Organic matter incorporation affects mechanical properties of soil aggregates. Soil Till. Res., 31:263-175, 1994. 